\title{
An Explicit Research on LENSCAM
}

\author{
Suhil Shavali, Sophiya Shaheen, Bibi Hajira, Syeda Ghousiya Rameez, Ume Salma S, Srikanth T \\ N, Azeem Ali
}

\begin{abstract}
The world of internet today has become a paralle form of life and living. Public are now capable of doing things which were not imaginable few years ago. Internet as enabled the use of website communication, email and a lot of anytime, anywhere IT solutions for the betterment of humankind.

We pursue life style innovation. The very first product of us "LENSCAM" is the tool that innovate human experiences about photo shooting. We want to help people to collect authentic moments with "LENSCAM" that never let the moment pass in life and contribute human life wealth. We find FUN in life, develop it by small essence of TECH and realize in the society with ART .The principle of our business is the conjoined three elements, FUN, ART and TECH. We believe Tech is something contributes to human life but tech itself never be realized. It should be found from FUN stuff. And any products or services should be aesthetically valuable to customers and the society.
\end{abstract}

Index term : Area-efficient, Low power, CSLA, Binary to excess one converter, Multiplexer

\section{INTRODUCTION}

The LENSCAM has a wink sensor built-in at the back of the device, which detects the movement of the muscles that run from the corner of the eye to the ear. When you blink naturally, the muscles of your face hardly move at all . However, when you wink, the muscles make a much bigger movement.

The LENSCAM sensor detects this type of movement. Hence, when winking, it can be much easier for the LENSCAM to detect the movement if you ensure that the area between the eye and the ear shows a strong movement. The LENSCAM cannot detect your winking actions if it is placed on top of the ear. Make sure not to attach the LENSCAM over the ear. The wink sensor detects the movement of the muscles that run from the corner of the eye to the ear. First, try winking normally. If the LENSCAM does not respond, try winking again by making sure to move the skin and muscles between the corner of the eye and your ear. As long as your skin moves when you wink, there is no need for you to wink for a long time.

\section{DELAY AND AREA EVALUATION OF THE BASICADDER BLOCKS}

To excess- 1 code converters (BEC) to improve the speed of addition. This logic can be implemented with any type of adder to further improve the speed. Using Binary to Excess1 Converter (BEC) instead of RCA in the regular CSLA we

Revised Manuscript Received on December 30, 2019.

Suhil Shavali, (Email: suhilshavali34@gmail.com)

Sophiya Shaheen, (Email: hbsophiya@gmail.com)

Bibi Hajira, (Email: hajiramussu@gmail.com)

Syeda Ghousiya Rameez, (Email: syedasiya26@ gmail.com)

Ume Salma S, (Email: alishasalma1123@gmail.com)

Srikanth T N, (Email: srikanthtn@gmail.com)

Azeem Ali, (Email: azeemali.royallad.cr7@gmail.com) can achieve lower area and power consumption. The main advantage of this BEC logic comes from the lesser number of logic gates than the Full Adder (FA) structure. And area equal to 1 unit. Wethen add up the number of gates in the longest path of a logic blockthat contributes to the maximum delay. The area evaluation is done bycounting the total number of AOI gates required for each logic block.

Based on this approach, the CSLA adder blocks of $2: 1$ mux, Half Adder(HA), and FA are evaluated and listed in Table I.

This article is an attempt to provide an understanding of

1) LENSCAM

2) OBJECTIVES OF LENSCAM

3) TYPES OF GLASS

4) Information of the person who involve in NEW TECH

\section{5)METHODOLOGY USED TO OPERATE LENSCAM \\ 6) ADVANTAGES \\ 7) COST OF PRODUCTION \\ 8) LOCATION OF PRODUCT USED}

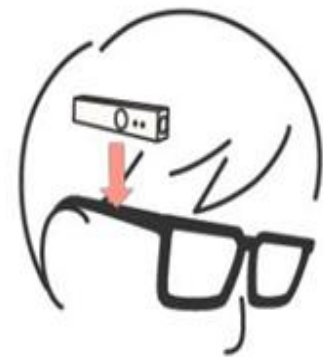

Attach to eye glasses

Because it attaches to any pair of eye glasses, you can save what you see, anytime anywhere

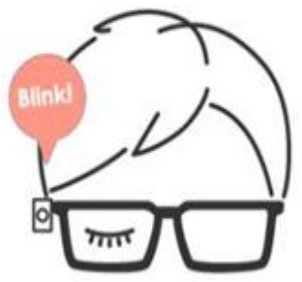

WINK to shutter

LENSCAM distinguishes strong wink or blink from natural ones so all you have to do is to WINK the shutter on your intention 


\section{An Explicit Research on LENSCAM}

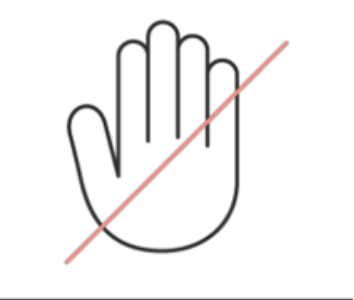

Hands Free

No need to use hands to take pictures. All you have to do is just to give a wink, or a blink

\section{Technology}

Size: $92.4 \mathrm{~mm} \times 17.6 \mathrm{~mm} \times 11.6 \mathrm{~mm}$

Weight: $25 \mathrm{~g}$

Memory: 8GB

Camera: HD CMOS 5 million pixels

Photos: 1.2 million pixels

Charging method: Micro USB Hours of

continuous use: approx. 1h 30mins

Charging time: approx. 1h 30mins Compatible smartphones: iPhone 5 or higher, iPod touch 5 th generation, iOS 8 or higher, and Android 4.3 or higher Blue light: Working normally.

Red light: Error. Turn off the device and restart it.

Yellow: Indicates that the internal USB memory card is being accessed.

Flashing light: Currently being charged

\section{Basic Structure Of Regular}

The camera shutter makes a noise when taking a picture. A startup sound is played when the power is turned on.

A shutdown sound is played when the power is turned off.

A sleep mode sound is played when entering sleep mode.

An active mode sound is played when returning from sleep mode.

A warning sound is played when there has been an error, or the battery is running low

The camera shutter makes a noise when taking a picture

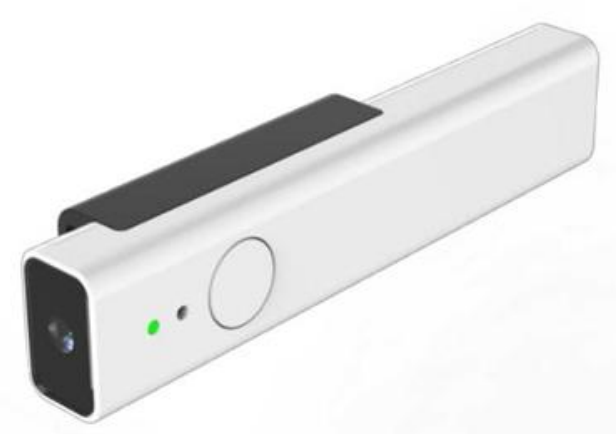

\section{METHOLOGY \{ USED PRODUCT DETAILS\}}

\section{- $\quad$ Turning the device on}

Make sure that the LENSCAM is charged sufficiently. To turn it on, press the round button on the side of the device for two seconds

- $\quad$ Pairing
Turn on Bluetooth on your smart phone. Download and open the LENSCAM app. You only need to establish a pairing the first time that you run the app. After this, the pairing is established automatically

\section{- $\quad$ Turning the device off}

To turn the device off, press the round button on the side of the LENSCAM for two seconds

\section{- Attachments}

The clip that attaches the device to your glasses is made of rubber and is easy to remove. Change the size of this attachment to fit the size of your glasses. Using the wrong attachment size may cause the device to fall off.

Also, the attachment sizes available may not be suitable if the temples on your glasses are extremely thin. In this case, please use some glasses with thicker temples

\section{- $\quad$ Taking pictures in dark places}

The LENSCAM is not designed to take pictures in dark places. Make sure to take photos in locations with as much light as possible. Also, the shutter speed is adjusted automatically depending on the light available, so pictures may look blurry if they are taken in dark places. We recommend stopping and staying still while taking a photo.

\section{- $\quad$ Auto-sleep}

The LENSCAM puts itself in sleep mode automatically after it has been inactive for more than three minutes. When you wink, the sleep mode is canceled. A wink that cancels the device's sleep mode also takes a picture; however, there is always a time lag between the wink and the photo being taken.

\section{- $\quad$ Auto-off}

The LENSCAM turns itself off automatically after 10 minutes of inactivity. To use the device after 10 minutes have passed, press the power button for two seconds to turn it on.

\section{ADVANTAGES\& RESULTS}

\section{Device response}

The LENSCAM has a wink sensor built-in at the back of the device, which detects the movement of the muscles that run from the corner of the eye to the ear. When you blink naturally, the muscles of your face hardly move at all; however, when you wink, the muscles make a much bigger movement. The LENSCAM sensor detects this type of movement. Hence, when winking, it can be much easier for the LENSCAM to detect the movement if you ensure that the area between the eye and the ear shows a strong movement

\section{Types of glasses}

There is no need for the LENSCAM sensor to be in contact with your skin. However, it does need to be placed within 1 centimeter $(0.4$ in) from your skin. The LENSCAM sensor may not respond if it ends up being far away from

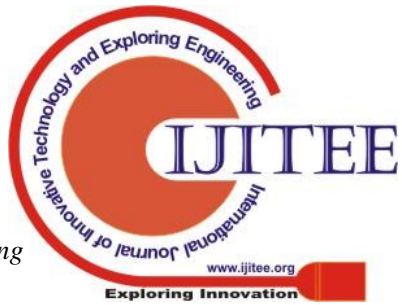


your skin, due to the curvature of the temples on your glasses, etc. Try changing to a pair of glasses that allows the LENSCAM to be placed within approximately 1 centimeter (0.4 in) from your face

\section{Brightness}

The brightness of the photos is adjusted based on the brightness of the area at the center of the images taken by the camera. If the central area is bright, the overall image becomes bright; and if the central object is dark, the rest of the image looks dark overall

\section{Focus}

The LENSCAM is configured to use pan focus, ensuring that the objects that are near and far are all relatively in focus. Hence, there is no need for you to adjust the focus on the device

\section{About Device}

The app is used by swiping on the photos that appear in the middle or on the square shaped like a photo

1. Swipe down to download photos from the LENSCAM device

2. Swipe right to delete a photo

3. Swipe left to save a photo. Photos are saved on your smartphone's camera roll

Tap on "SHARE" on the right bottom corner of the picture on the main screen. It will show file sharing menu that is defaulted to your smartphone. You will be able to send or share photos via e-mail, facebook, twitter and so on

\section{CONCLUSION}

With the spread of computers and internet, Technology has emerged as a major resource. The internet brings joy to our lives but at the same. The world of internet today has become a parallel form of life and living. Public are now capable of doing things which were not imaginable few years ago. Internet as enabled the use of website communication, email and a lot of anytime, anywhere IT solutions for the betterment of humankind. We

pursue life style innovation. The very first product of us "LENSCAM" is the tool that innovate human experiences about photo shooting. We want to help people to collect authentic moments with "LENSCAM" that never let the moment pass in life and contribute human life wealth. We find FUN in life, develop it by small essence of TECH and realize in the society with ART .The principle of our business is the conjoined three elements, FUN, ART and TECH. We believe Tech is something contributes to human life but tech itself never be realized. It should be found from FUN stuff. And any products or services should be aesthetically valuable to customers and the society.

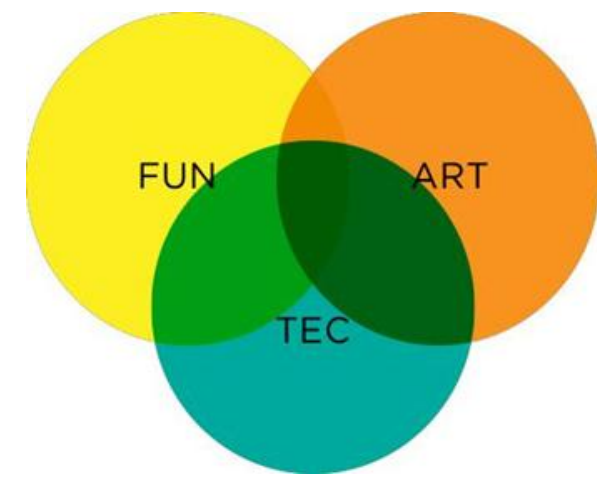

\section{REFERENCES}

1. N. Ardley, 101 great science experiments. DK publishings,2006

2. S. Ray, the Photographic lens. Focal press, oxford, 1992.

3. H. Gernsheim, a concise history of photography. DOVER publications, 1986.

4. J.petterchak, photography genius: GEORGE R LAWRENCE and "the hitherto impossible". Journal of the Illinois state historical society, summer 2002 